\title{
Dieta Cetogénica en el paciente con epilepsia refractaria
}

\author{
Ketogenic Diet in patients with refractory epilepsy
}

\author{
María J. Rebollo G. a, Ximena Díaz SM.c, Marcela Soto R. ${ }^{a}$, Johanna Pacheco A. ${ }^{\text {, }}$, \\ Scarlet Witting E. ${ }^{\text {,dd }}$, Isidora Daroch R. ${ }^{c}$, Francisco Moraga M. ${ }^{a}$
}

\author{
aServicio de Pediatría, Unidad de Nutrición, Hospital San Borja Arriarán. Santiago, Chile \\ bervicio de Neuropsiquiatría Infantil, Hospital San Borja Arriarán. Santiago, Chile \\ ‘Departamento de Nutrición, Hospital San Borja Arriarán. Santiago, Chile \\ dDepartamento de Pediatría Campus Central, Universidad de Chile. Santiago, Chile
}

Recibido: 16 de diciembre de 2019; Aceptado: 7 de junio de 2020

¿Qué se sabe del tema que trata este estudio?

La dieta cetogénica (DC) es un tratamiento no farmacológico efectivo para el manejo de la epilepsia refractaria (ER) en población pediátrica, pudiendo reducir y abolir las crisis convulsivas, así como reducir el uso de fármacos antiepilépticos.

\section{¿Qué aporta este estudio a lo ya conocido?}

Aporta una experiencia nacional de DC en ER en con un número importante de casos pediátricos manejados con protocolos de dieta cetogénica elegidos para cada situación clínica, con buena respuesta en el control de crisis epilépticas y sin compromiso nutricional importante.

\section{Resumen}

La epilepsia afecta a 0,5 a $1 \%$ de la población. El $25 \%$ de los niños que la presentan tienen epilepsia refractaria (ER) a fármacos antiepilépticos (FAE) y en ellos la dieta cetogénica (DC) surge como un tratamiento no farmacológico efectivo. Objetivo: Describir el impacto de la DC en la frecuencia de crisis epilépticas y el estado nutricional en pacientes pediátricos con ER. Pacientes y Método: Se revisaron registros médicos de los pacientes con ER que recibieron DC entre los años 2008 y 2018 registrando variables: edad, diagnóstico, número de crisis, número de FAE, respuesta y complicaciones. La DC se inició en todos los casos estando hospitalizado durante un período no mayor a siete días, con evaluación nutricional antropométrica con medición de peso y talla. Resultados: Se analizaron 35 DC en 33 pacientes. La mediana de edad al inicio fue 4,8 años (RIC 2,3-6,8 años). Se utilizó DC clásica en $49 \%$ de los pacientes, Dieta Atkins Modificada en 37\% y Dieta de Bajo Índice Glicémico en $14 \%$. Su promedio de duración fue 13 meses +11 DS meses. Luego de tres meses de iniciada la DC, la reducción de al menos un 50\% de las crisis se observó en $82 \%$ de los casos, y $20 \%$ quedó libre de crisis. Se registraron efectos secundarios en 21 pacientes, la mayoría gastrointestinales $(62 \%)$ y dislipidemia (14\%). No hubo compromiso de la talla. Todos los efectos secundarios se resolvieron con manejo médico. Conclusiones: La DC es un tratamiento efectivo en pacientes pediátricos con ER. Las complicaciones asociadas fueron fácilmente controladas por un equipo multidisciplinario, sin impacto nutricional importante.

Correspondencia:

Francisco Moraga M.

fjmoraga@gmail.com 


\section{Abstract}

Epilepsy affects 0.5 to $1 \%$ of the population. $25 \%$ of pediatric patients have drug-resistant epilepsy (DRE). Ketogenic Diet (KD) emerges as an effective, non-pharmacological treatment in this group. Objective: To describe the effect of $\mathrm{KD}$ on seizure control and nutritional status in children whit DRE. Patients and Method: We reviewed the medical records of patients with DRE treated with KD, between 2008 and 2018, evaluating age, diagnosis, number of seizures, number of antiepileptic drugs used, clinical outcomes, and complications. The KD was initiated in all patients hospitalized for a period no longer than seven days, who were evaluated for their nutritional and anthropometric status, with weight and height measurements according to the clinical condition. Results: We analyzed $35 \mathrm{KD}$ in 33 cases. The median age of KD initiation was 4.8 years with an interquartile range (IQR) of 2-3 to 6.8 years. Classical KD was used in $49 \%$ of patients, Modified Atkins Diet (MAD) in 37\%, and Low-Glycemic Index Treatment (LGIT) in 14\% of cases. The average duration was 13 months (SD 11 months). After three months of using $\mathrm{KD}$, we observed at least $50 \%$ reduction of seizures in $82 \%(27 / 33)$ of the patients, out of these, $22.8 \%$ presented $90 \%$ or more reduction of seizures, and $20 \%$ ended up seizure-free. Adverse events were observed in 21 patients, mainly gastrointestinal $(62 \%)$ and dyslipidemia (14\%), without effect on height. All side effects resolved with medical management. Conclusions: KD is a useful treatment in pediatric patients with DRE without nutritional impact. The adverse events were easily controlled if the patients are evaluated by a multidisciplinary team, according to international guidelines.

\section{Keywords:}

Ketogenic Diet;

Refractory Epilepsy;

Atkins Diet;

Glycemic Index;

Dyslipidemia

\section{Introducción}

La epilepsia es una entidad clínica que afecta al $0,5 \%$ a $1 \%$ de la población ${ }^{1}$. El $60 \%$ de los casos se inicia durante la infancia ${ }^{1}$ y alrededor del $25 \%$ de los pacientes presenta una epilepsia refractaria $(E R)^{2,3}$, definida como un inadecuado control de las convulsiones a pesar del tratamiento con al menos dos fármacos antiepilépticos (FAE), a dosis adecuadas y durante un tiempo adecuado, condicionando un deterioro de la función neurológica y un empeoramiento en la calidad de vida ${ }^{4-6}$. En estos pacientes se plantea adicionar dieta cetogénica (DC) al tratamiento farmacológico ${ }^{7,8}$. La DC consiste en una alimentación alta en grasas y baja en carbohidratos diseñada para simular los efectos bioquímicos del ayuno ${ }^{3,8}$.

Existen varias modalidades de DC, de las cuales cuatro tipos cuentan con evidencia que apoyan su uso en $\mathrm{ER}^{8,9}$. La DC clásica (DCC), que proporciona el 90\% de las calorías como grasa con uso de triglicéridos de cadena larga (TCL) y el 10\% de las calorías restantes como proteínas e hidratos de carbono, estableciendo una relación calórica entre lípidos y la suma de proteínas más carbohidratos de 4:1 o 3:1. Otra es la DC con triglicéridos de cadena media (DTCM), que produce mayor cantidad de cetonas por gramo de grasa y proporciona $60 \%$ de las calorías como grasas, pudiendo ser la mitad de ellas triglicéridos de cadena media (porcentaje que varía según tolerancia), manteniendo relación calórica de 3:1. También se usa la DC Atkins modificada (DAM), menos estricta que las anteriores, que aporta una cantidad fija de hidratos de carbono de $10-15 \mathrm{~g} / \mathrm{d}$ en niños y $20 \mathrm{~g} / \mathrm{d}$ en adultos, con aporte calórico de origen proteico de 20\%-30\% y aporte calórico dado por grasas de $60-70 \%$. Finalmente, la DC con bajo índice glicémico (DBIG), que proporciona 30-40 g de hidratos de carbono, con índice glicémico bajo 50 y aporte de 50\%-60\% de las calorías como grasa'.

El mecanismo por el cual la DC ejercería su efecto anticonvulsivante no está claramente dilucidado. Se han descrito numerosas hipótesis sobre cómo actúa, pero ninguno ha sido ampliamente aceptado como único mecanismo responsable de su acción. Probablemente se trate de diferentes mecanismos de acción que actúan en paralelo y sinérgicamente $e^{10-12}$.

Dado que la DC implica un aporte deficiente de micronutrientes, es indispensable la suplementación con vitaminas, minerales y elementos traza ${ }^{9,13}$ y considerando que la DC utiliza grasa como principal fuente de energía, cualquier error innato que comprometa el metabolismo de los lípidos constituye una contraindicación para su realización ${ }^{14-16}$.

Varios estudios describen que la correcta utilización de DC en sujetos con ER disminuye al menos un $50 \%$ el número de crisis en la mitad de los pacientes tratados y una reducción de más del $90 \%$ de las convulsiones en un tercio de ellos ${ }^{12,16-21}$.

El objetivo de esta investigación fue describir el impacto de la DC en la frecuencia de crisis epilépticas y el estado nutricional en pacientes pediátricos con ER. El análisis de este grupo de pacientes en relación con el impacto de la DC en la disminución de las crisis epilépticas de acuerdo con la etiología de la epilepsia y tipo de crisis epilépticas se reportó en otra publicación ${ }^{22}$. 


\section{Pacientes y Método}

\section{Diseño}

Se revisaron los registros médicos de todos los pacientes derivados desde el Servicio de Neurología Infantil al equipo de Nutrición infantil del Hospital Clínico San Borja Arriarán (HCSBA), para tratamiento de ER con DC durante el período comprendido entre los años 2008 y 2018.

Una vez indicada la DC por parte del equipo de Neurología, el equipo de Nutrición evaluó la condición del paciente y su familia para ver la factibilidad de su realización y valorar adherencia, entregando información a padres y cuidadores.

\section{Pacientes y protocolo de DC}

Los pacientes fueron analizados por sexo, diagnóstico, vía de alimentación, número de FAE utilizados y número de crisis/día referidas por el cuidador al momento de inicio de la DC. Se incluyeron dos casos cuya presentación clínica fue con estatus epiléptico, lo que implicó que no fue posible cuantificar el número de crisis.

La DC se inició en todos los casos con el paciente hospitalizado durante un período no mayor a siete días, en el cual se realizó evaluación nutricional antropométrica con medición de peso y talla según condición clínica. Para este análisis en 17 casos se usó curvas específicas para pacientes con parálisis cerebral ${ }^{23}$, registrando IMC/E y T/E medido en percentiles y considerando desnutrición a pacientes con IMC/E menor a Pc 5. En los casos restantes, se usó estándares antropométricos OMS $2006^{24}$ para los menores de 5 años, registrando $\mathrm{P} / \mathrm{T}$ y $\mathrm{T} / \mathrm{E}$ con medición de puntaje $\mathrm{Z}$ y OMS $2007^{25}$ para los mayores de 5 años, registrando IMC/E y T/E con medición de puntaje Z. En pacientes analizados con curvas OMS se usaron los puntos de corte habituales para el diagnóstico nutricional. Se realizaron exámenes de laboratorio de ingreso según protocolo de estudio (figura 1) y se completó un plan de educación al cuidador.

El tipo de DC se escogió considerando la vía de alimentación de los pacientes y la situación clínica de cada uno. En aquellos que se alimentaban por vía oral, se prefirió utilizar modalidades DAM y BIG lo que permite mejorar la adherencia y la tolerancia debido a su mejor palatabilidad. En pacientes alimentados por vía enteral a través de gastrostomía (GTT), se prefirió la DCC 3:1 o 4:1.

La DC se inició sin período de ayuno ni restricción hídrica y fue instaurada desde el comienzo con el aporte calórico total, calculado de acuerdo con sus requerimientos. La razón cetogénica se fue aumentando progresivamente hasta lograr el objetivo de cetosis al tercer día. Antes del año 2015 se midió cetosis me- diante cetonuria, considerando como valor positivo la presencia de 3 cruces, equivalente a $28 \mathrm{mmol} / \mathrm{L}$. Desde el año 2015, la medición de cetosis se realizó con determinación de Betahidroxibutirato en muestra venosa, considerando como positivo un valor mayor o igual a $2 \mathrm{mmol} / \mathrm{L}$. Posteriormente, el control de cetosis fue realizado según protocolo ambulatorio como muestra la figura 1.

En los dos casos con estatus epiléptico, se inició la DC con el paciente monitorizado en Unidad de Cuidados Intensivos. En ambos se usó DC clásica 4:1 vía enteral a través de SNG y su implementación no varió con relación a lo descrito para los otros pacientes.

Todos los pacientes fueron dados de alta con un set de minutas elaboradas en nuestro centro, personalizadas y estrictamente calculadas considerando para tal efecto la Tabla de Composición de Alimentos del Instituto de Nutrición y Tecnología de los Alimentos de la Universidad de Chile (INTA) ${ }^{26}$.

En preescolares con DAM se usó un porcentaje de calorías proteicas más bajo que el recomendado ${ }^{27,28}$, con el fin de entregar una alimentación con un aporte proteico más adecuado para la edad, por lo que se usó

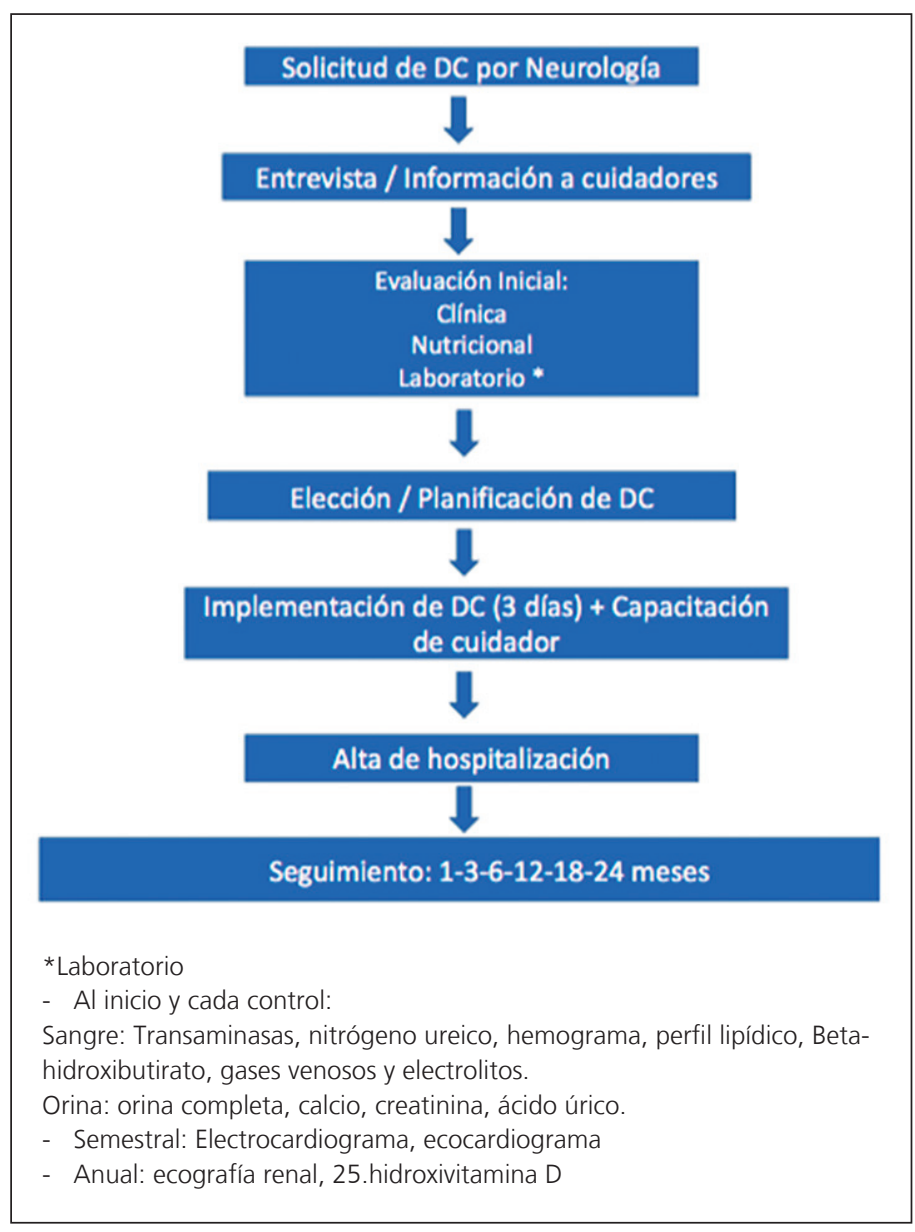

Figura 1. Protocolo de Dieta Cetogénica aplicado a la población de estudio. 
un $\mathrm{P} \%$ de 15, manteniendo el aporte de hidratos de carbono mencionado en otras publicaciones ${ }^{27-29}$.

Los lípidos fueron planificados en base a una cantidad fija de aceite rico en omega $3(10 \mathrm{ml} / \mathrm{d})$ y la diferencia se completó en base a porcentajes equivalentes de aceite de oliva y aceite de maravilla, con el propósito de incorporar a la dieta ácidos grasos monoinsaturados y poliinsaturados, para lograr una proporción lo más cercana posible a $1: 1: 1^{27,29}$.

Todos los pacientes fueron suplementados con micronutrientes y calcio acorde a requerimientos (RDI). Dentro de la minuta entregada se incluyó ingesta de huevo para asegurar un aporte apropiado de selenio ${ }^{26}$. Se administró vitamina D3 en dosis iniciales de 1.000 UI/d, la que se ajustó en base a las concentraciones séricas de $25 \mathrm{OH}$ vitamina $\mathrm{D}$ cuando ésta estuvo disponible. Los pacientes que recibían tratamiento con ácido valproico fueron suplementados con L-Carnitina en dosis de $50 \mathrm{mg} / \mathrm{kg} / \mathrm{d}$.

La DC se mantuvo por un periodo de 24 meses en caso de éxito y un máximo de 3 meses en caso de fracaso. En ambas situaciones, el retiro de la dieta se realizó en forma paulatina durante 4 semanas. El seguimiento ambulatorio se realizó en Policlínico de Nutrición con controles a los 1, 3, 6, 12, 18 y 24 meses de iniciada la dieta. En cada control se realizó evaluación antropométrica y adecuación del aporte calórico, con variación de minutas cuando fue necesario, solicitud de exámenes de acuerdo con protocolo (que incluyó medición de Betahidroxibutirato en laboratorio de nuestro hospital), pesquisa de complicaciones y control clínico, incluyendo la información entregada por el cuidador con relación al número de crisis.

Se consideró como respuesta favorable una reducción del número de crisis mayor o igual al 50\% ${ }^{12,29}$. Dislipidemia fue definida según los puntos de corte para lípidos sanguíneos sugeridos por panel de expertos AAP en 1992 y complementados en el año 2011 para valores de triglicéridos (TG) y colesterol HDL (CHDL $)^{30-32}$.

\section{Análisis estadístico}

Se resumieron los resultados en una base de datos en el programa Excel. El análisis estadístico se desarrolló con el programa GraphPad Prism 8, las variables cuantitativas fueron evaluadas con mediana y rango intercuartílico (RIC) o promedio, desviación estándar y porcentajes. Se usó el Test de Wilcoxon para muestras pareadas para el análisis del porcentaje de disminución de crisis (figura 2).

\section{Ética}

Este trabajo fue presentado y aprobado por el Comité Ético Científico del Servicio de Salud Metropolitano Central, Santiago con fecha 09-10-2019.

\section{Resultados}

Se analizaron 35 DC en 33 pacientes con mediana de edad al inicio de la DC de 4,8 años y RIC de 2,3-6,8 años, variando entre un mínimo de 4 meses y un máximo de 15 años.

En dos pacientes hubo abandono de la dieta, la que fue posteriormente reinstaurada por indicación de Neurología. Las características generales de las DC efectuadas, así como su distribución según diagnóstico, se presentan en la tabla 1. La distribución de pacientes según tipo de DC utilizada se presenta en la tabla 2.

El estado nutricional al ingreso al protocolo fue 22 (63\%) eutrófico, 3 (8,5\%) riesgo de desnutrición, 2 $(5,7 \%)$ desnutrición, $2(5,7 \%)$ sobrepeso y $6(17,1 \%)$ obeso. Al momento de retirarse del protocolo la distribución fue $20(57,2 \%)$ eutrófico, $4(11,5 \%)$ riesgo de desnutrición, 3 (8,5\%) desnutrición, 6 (17\%) sobrepeso y $2(5,7 \%)$ obeso. Al comparar los pacientes consigo mismo al ingreso y al término de la dieta, $23(65,7 \%)$ permanecieron sin cambios, $2(5,7 \%)$ que ingresaron en riesgo de desnutrición se desnutrieron, 2 (5,7\%) que ingresaron eutróficos pasaron a estar en riesgo de desnutrición y $2(5,7 \%)$ que ingresaron eutróficos terminaron en sobrepeso. Seis pacientes $(17,1 \%)$ mejoraron su antropometría después de haber iniciado con exceso o déficit de peso. En aquellos pacientes que se mantuvieron en DC durante un periodo de al menos seis meses, no se deterioró la talla.

De las 35 DC realizadas, catorce (40\%) completaron nuestro protocolo. Diez pacientes (28\%) se suspendieron dentro de los primeros 3 meses (seis por no presentar respuesta y cuatro por abandono de la dieta). De los once restantes, en solo un paciente se suspendió la dieta por complicaciones (cuadro diarreico a repeti-

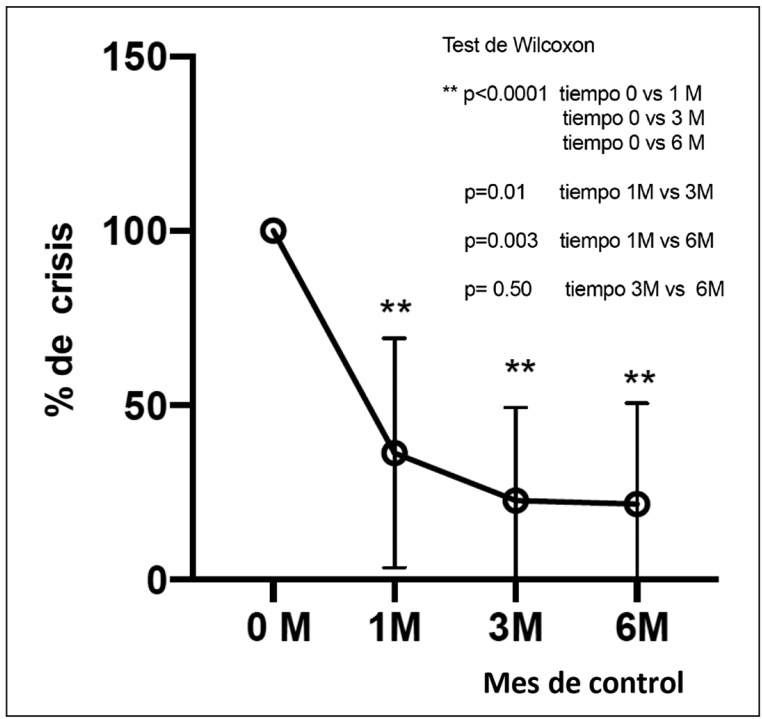

Figura 2. Porcentaje de reducción de crisis epilépticas en el tiempo. 
Tabla 1. Características de la población

\begin{tabular}{|c|c|c|}
\hline Variable & Categorización & Total (n) \\
\hline Sexo & $\begin{array}{l}\text { Femenino } \\
\text { Masculino }\end{array}$ & $\begin{array}{l}19 \\
16\end{array}$ \\
\hline Número de FAE & $\begin{array}{l}\leq 2 \\
3 \text { a } 4 \\
\geq 5\end{array}$ & $\begin{array}{r}5 \\
28 \\
2\end{array}$ \\
\hline Número de crisis/día & $\begin{array}{c}<5 \\
5 \text { a } 10 \\
11 \text { a } 30 \\
\text { Estatus }\end{array}$ & $\begin{array}{r}5 \\
19 \\
9 \\
2\end{array}$ \\
\hline Vía de alimentación & $\begin{array}{l}\text { Enteral (GTT) } \\
\text { Oral }\end{array}$ & $\begin{array}{l}14 \\
21\end{array}$ \\
\hline Diagnóstico etiológico desconocido* & & 17 \\
\hline Síndrome clínico & $\begin{array}{l}\text { Síndrome de West } \\
\text { Síndrome de Lennox Gastaut } \\
\text { Síndrome de Ohtahara } \\
\text { Síndrome de Dosse } \\
\text { Síndrome de Rett }\end{array}$ & $\begin{array}{l}4 \\
1 \\
1 \\
2 \\
1\end{array}$ \\
\hline Genético & $\begin{array}{l}\text { Síndrome de Dravet } \\
\text { Déficit de Glut } 1\end{array}$ & $\begin{array}{l}2 \\
1\end{array}$ \\
\hline Estructural metabólico & $\begin{array}{l}\text { Esclerosis tuberosa } \\
\text { Encefalopatía herpética }\end{array}$ & $\begin{array}{l}3 \\
3\end{array}$ \\
\hline
\end{tabular}

FAE: Fármacos antiepilépticos. GTT: gastrostomía. *Corresponde a 17 DC en 15 pacientes.

Tabla 2. Tipo de dieta cetogénica (DC) realizada en la población de estudio

\begin{tabular}{|c|c|c|c|c|c|c|}
\hline \multirow{2}{*}{$\begin{array}{l}\text { Tipo de DC } \\
\text { Clásicas }\end{array}$} & \multicolumn{2}{|c|}{ Total de DC } & \multicolumn{3}{|c|}{ Porcentaje calórico de macronutrientes } & \multirow[t]{2}{*}{ Razón cetogénica } \\
\hline & $n$ & $\%$ & $\%$ G & $\% \mathrm{P}$ & $\% \mathrm{CHO}$ & \\
\hline $4: 1$ & 12 & 35 & 90 & 6 & 4 & $4: 1$ \\
\hline $3: 1$ & 5 & 14 & 85 a 87 & 8 a 10 & 4 a 7 & $3: 1$ \\
\hline Atkins modificada* & 13 & 37 & 50 a 79 & 15 a 30 & 5 a 20 & $1,5: 1$ \\
\hline Bajo índice glicémico & 5 & 14 & 60 a 66 & 17 a 27 & 13 a 23 & $1,5: 1$ \\
\hline
\end{tabular}

\%G: Porcentaje calórico proveniente de grasas. \%P: Porcentaje calórico proveniente de proteínas. \%CHO: Porcentaje calórico proveniente de carbohidratos. ${ }^{*}$ Corresponde a 13 DC efectuadas en 11 pacientes.

ción) y los demás se retiraron antes de lo programado por dificultades en la adherencia.

Luego de 3 meses de iniciado el protocolo, solo seis casos no presentaron buena respuesta: cuatro de ellos disminuyeron su frecuencia de crisis y los otros dos disminuyeron sus crisis en menos del 50\%. Los 29 casos restantes presentaron una respuesta favorable, es decir, una disminución del $50 \%$ o más del número de crisis, con la siguiente distribución: catorce disminuyeron sus crisis entre 50 y $90 \%$, ocho lo hicieron en más del $90 \%$ y siete evolucionaron con remisión total de las crisis, lo que se observa en la tabla 3. Destaca que los dos pacientes en estatus epiléptico también tuvieron una respuesta favorable.

En la figura 2 se muestra la eficacia clínica de la DC, expresada como el porcentaje de disminución de crisis observado entre el inicio de la dieta y luego de 1, 3 y 6 meses de instaurada. Esta disminución fue estadísticamente significativa.

Tabla 3. Porcentaje de reducción de crisis epilépticas

\begin{tabular}{lcc}
\hline \% Reducción de crisis & N & Porcentaje (\%) \\
\hline Sin reducción de crisis & 4 & 11,4 \\
Menor $50 \%$ & 2 & 5,7 \\
$50-90 \%$ & 14 & 40 \\
Mayor 90\% & 8 & 22,8 \\
$100 \%$ & 7 & 20 \\
\hline
\end{tabular}


En nuestra serie observamos que 21 pacientes presentaron complicaciones, siendo la mayoría (13 casos) gastrointestinales: once casos de constipación, todos manejados satisfactoriamente con polietilenglicol (PEG) a dosis habituales; un lactante de nueve meses que presentó varios episodios de diarrea requiriendo manejo hospitalizado y un paciente con vómitos a repetición cuyo cuadro cedió luego de cambiar su DCC 4:1 por una DC tipo DAM. En relación al resto de complicaciones observadas, dos pacientes usuarios de Topiramato presentaron acidosis metabólica leve de resolución espontanea, sin requerir uso de bicarbonato y sin repercusión clínica. Tres pacientes presentaron litiasis renal que fue resuelta médicamente por el equipo de Nefrología mediante el uso de citrato de potasio. Otros tres pacientes presentaron alteración en el perfil lipídico, los tres menores de 10 años y de presentación precoz, pesquisado en el control de los 3 meses. En dos de ellos se elevó tanto el colesterol total (CT) como los triglicéridos (TG), el primero con CT de $210 \mathrm{mg} / \mathrm{dl} \mathrm{y}$ TG de 137 mg/dl y el segundo con CT de $263 \mathrm{mg} / \mathrm{dl}$ y TG de $166 \mathrm{mg} / \mathrm{dl}$. El tercer paciente con alteración de lípidos plasmáticos presentó solo elevación del CT de $246 \mathrm{mg} / \mathrm{dl}$. Todos fueron tratados con medidas dietéticas, logrando niveles plasmáticos de CT menor de $200 \mathrm{mg} / \mathrm{dl}$ y TG menor de $100 \mathrm{mg} / \mathrm{dl}$ disminuyendo así su nivel de riesgo según puntos de corte de consenso internacional ${ }^{30-32}$. Otros 3 pacientes presentaban al momento de inicio de la dieta CT sobre $200 \mathrm{mg} / \mathrm{dl} \mathrm{y}$ en todos ellos, con el ajuste dietético, se llegó a niveles plasmáticos de CT menor a $200 \mathrm{mg} / \mathrm{dl}$.

\section{Discusión}

La DC, como parte del tratamiento para ER en niños, se encuentra ampliamente validada en la literatura, con una eficacia que varía entre $56 \%$ y $85 \%$ en la reducción de más del $50 \%$ de las crisis y logrando una remisión de éstas en un 15\% a 55\% de los pacientes ${ }^{11,12}$. Esta variabilidad podría tener relación con los diagnósticos de base, ya que existirían condiciones neurológicas en las cuales se ha reportado mejor respuesta ${ }^{4,5,29}$.

En nuestra experiencia, en el 82,8\% de los casos obtuvimos una respuesta favorable, definida como la reducción de la crisis mayor al 50\%, destacando que un $20 \%$ de los pacientes presentó una remisión total de las crisis. Una posible explicación para estos resultados podría deberse a que, de nuestros 18 casos con diagnóstico sindromático conocido, un gran porcentaje pertenece al grupo señalado de síndromes y condiciones asociados con mayor probabilidad de respuesta exitosa a $\mathrm{DC}^{4,5,29}$. Solo en 5 pacientes se pudo contar con diagnóstico molecular para la determinación de mutaciones genéticas asociadas, de los cuales 2 presen- taban la mutación SCN1A, una de las que se asocia a mejor respuesta ${ }^{33}$. A futuro, contar con esta herramienta diagnóstica podría ayudarnos a evaluar respuesta y favorecer la selección de pacientes candidatos a DC.

Otro elemento para considerar y que se repite en otros reportes con respuesta favorable, es la educación a los cuidadores ${ }^{29}$. En nuestro centro, durante la hospitalización, se realiza un taller intensivo teórico práctico para educar a los cuidadores y asegurar instancias para que consulten ante cualquier duda.

En dos de los pacientes en que se usó DCC 4:1 hubo disminución de las crisis pese a no haberse demostrado cetonemia (medido con Betahidroxibutirato durante la hospitalización). En ambos casos existió uso de fármacos que interfieren con el metabolismo de la gluco$\mathrm{sa}^{34,35}$ : una paciente con panhipopituitarismo usuaria de hormona de crecimiento y un paciente con cuadros obstructivos recurrentes recibiendo budesonida inhalatoria, que al suspenderse alcanzó cetosis. Una posible explicación es que se ha propuesto que la restricción de glucosa jugaría un rol en el efecto anticonvulsivante, lo que queda demostrado con la efectividad de las dietas de bajo índice glicémico ${ }^{34,35}$.

Gran parte de los cuidadores reportaron mejoría en el estado de conciencia de los pacientes, lo cual fue una motivación adicional a seguir con la dieta. Aunque este hallazgo no formaba parte de nuestro análisis, permite establecer una nueva línea de trabajo evaluando el bienestar subjetivo del paciente y de sus cuidadores. Ya en el año 2006, Fasarat y Kossoff demostraron que alcanzar o superar las expectativas de los padres en cuanto a la mejoría cognitiva, se correlacionó significativamente con una mayor duración de la dieta ${ }^{36}$.

Desde el año 2008 hay publicaciones del uso de DC en pacientes con diagnóstico de estatus epiléptico y que presentaron buena evolución ${ }^{37}$. En nuestra experiencia, los dos pacientes con esta condición respondieron favorablemente. En la literatura reciente, se describe el uso de DC vía parenteral en este tipo de casos, lo que nos parece interesante considerar para su posible uso a futuro ${ }^{38,39}$.

En cuanto al porcentaje de abandono de la DC, nuestra experiencia es similar a lo descrito en otros estudios $^{29,40-42}$. A los 6 meses, 13 de los 35 casos (37\%) abandonaron la dieta, la mayoría por transgresión de las minutas. En este grupo se encuentran principalmente aquellos niños que deambulan, se alimentan vía oral y tienen acceso autónomo a alimentos distintos a los contenidos en su pauta diaria de alimentación.

En la literatura se describen varios efectos adversos a corto y largo plazo como consecuencia del alto contenido en grasas y el bajo aporte de carbohidratos, fibra y micronutrientes ${ }^{29,43,44}$. Para disminuir el riesgo de deficiencias nutricionales, actualmente y en pacientes menores de dos años, usamos formulas comerciales es- 
pecíficas dado que se encuentran en un período crítico de crecimiento.

En nuestra serie los eventos adversos más frecuentes fueron gastrointestinales, siendo la constipación la más descrita (31\%). Sin embargo, solo una de las DC realizadas debió suspenderse a causa de un evento adverso: lactante de nueve meses recibiendo DCC 3:1 y que presentó múltiples hospitalizaciones asociadas a diarrea y alteraciones hidroelectrolíticas.

Nuestra baja incidencia de dislipidemias $(8,5 \%)$ en comparación con lo reportado en otras publicaciones (14\%-59\%) podría explicarse porque en todos los casos se buscó obtener una relación equilibrada de lípidos, optimizando el aporte de ácidos grasos poliinsaturados con una cantidad fija de omega $3^{29,45}$. Adicionalmente, algunos estudios que evalúan distintas distribuciones de los tipos de grasas muestran que una mayor proporción de ácidos grasos poliinsaturados favorecerían la presencia de cetosis ${ }^{46-48}$.

En relación con el impacto en el crecimiento, existe controversia en la literatura, reportándose, en algunos casos, desaceleración de talla ${ }^{29,44,49-53}$. Ninguno de nuestros pacientes con al menos 6 meses de tratamiento presentó deterioro de la talla durante la realización de la DC. Una posible explicación es que no restringimos el aporte de energía. Nos parece de alto interés evaluar a futuro la relación entre DC y crecimiento, considerando patología de base, factores nutricionales, endocrinos y metabólicos que pudieran afectar el patrón de crecimiento.

En conclusión, los resultados obtenidos en nuestra experiencia muestran un éxito terapéutico similar al descrito en centros expertos ${ }^{29}$, validando a nivel local la adición de DC al tratamiento farmacológico de la ER en la edad pediátrica. Nuestro trabajo muestra la evolución de un número importante de pacientes, bajo un protocolo de seguimiento establecido, en donde se adecuó el tipo de dieta a cada paciente considernado sus caracteristicas clínicas, requerimientos y tolerancia alimentaria, lo que fue evaluado durante todo el periodo de tratamiento. Destaca que no hubo compromiso de la talla en los pacientes que usaron dieta más de tres meses. Esta experiencia deja abierta la posibilidad a otras áreas de investigación que permitan establecer el impacto de la DC no solo en el número de crisis, sino en la calidad de vida y bienestar del paciente y su entorno.

\section{Responsabilidades Éticas}

Protección de personas y animales: Los autores declaran que los procedimientos seguidos se conformaron a las normas éticas del comité de experimentación humana responsable y de acuerdo con la Asociación Médica Mundial y la Declaración de Helsinki.

Confidencialidad de los datos: Los autores declaran que han seguido los protocolos de su centro de trabajo sobre la publicación de datos de pacientes.

Derecho a la Privacidad y Consentimiento Informado: Los autores declaran que la información ha sido obtenida de datos previos en forma anonimizada, por lo cual el Comité de Ética de Investigación en uso de sus facultades, ha eximido de la obtención de un consentimiento informado, lo cual consta en el acta respectiva.

\section{Conflicto de intereses}

Los autores declaran no tener conflicto de intereses.

\section{Referencias}

1. Armeno M, Caraballo R, Vaccarezza $\mathrm{M}$, et al. Consenso nacional sobre dieta cetogénica. Rev Neurol 2014;59(5):21323.

2. Kossoff EH. Intractable childhood epilepsy: choosing between the treatments. Semin Pediatr Neurol 2011;18:145-9.

3. Freeman JM, Kossoff EH, Hartman AL. The ketogenic diet: one decade later. Pediatrics 2007;119(3):535-43.

4. Alberti M, Agustinho A, Argumedo L, et al. Recomendaciones para el manejo clínico pediátrico de la dieta cetogénica en el tratamiento de la epilepsia refractaria. Arch Argent Pediatr 2016;114(1):56-63.

5. Pablos-Sánchez T, Oliveros-Leal L, Núñez-Enamorado N, et al. Experiencia en el tratamiento con dieta cetogénica de la epilepsia refractaria en la edad pediátrica. Rev Neurol 2014;58:55-62.

6. Kwan P, Arzimanoglou A, Berg AT, et al. Definition of drug resistant epilepsy: consensus proposal by the ad hoc Task Force of the ILAE Commission on Therapeutic Strategies. Epilepsia 2010;51:1069-77.

7. Caraballo RH, Vining E. Ketogenic diet. Handb Clin Neurol 2012;108:783-93.

8. Neal EG, Chaffe HM, Schwartz RH, et al. The ketogenic diet in the treatment of epilepsy in children: a randomised, controlled trial. Lancet Neurol 2008;7:500-6.

9. Winesett SP, Bessone SK, Kossoff EH. The ketogenic diet in pharmacoresistant childhood epilepsy. Expert Rev Neurother 2015;15(6):621-8.
10. Rho JM. How does the ketogenic diet induce anti-seizure effects? Neurosci Lett 2017;637:4-10.

11. Youngson N, Morris M, Ballard J. The mechanisms mediating the antiepileptic effects of the ketogenic diet, and potential opportunities for improvement with metabolism-altering drugs. Seizure 2017;52:15-9.

12. Roehl K, Sewak SL. Practice Paper of the Academy of Nutrition and Dietetics: Classic and Modified Ketogenic Diets for Treatment of Epilepsy. J Acad Nutr Diet 2017;117:1279-92.

13. Tagliabue A, Bertoli S, Trentani C, Borrelli P, Veggiotti P. Effects of the ketogenic diet on nutritional status, resting energy expenditure, and substrate oxidation in patients with medically refractory epilepsy: a 6-month prospective 
observational study. Clin Nutr 2012;31:246-9.

14. Kossoff EH, Rowley H, Sinha SR, Vining EP. A prospective study of the modified Atkins diet for intractable epilepsy in adults. Epilepsia 2008;49:316-9.

15. Kossoff EH, Laux LC, Blackford R, et al. When do seizures improve with the ketogenic diet? Epilepsia 2008;49:329-33.

16. Kang HC, Lee YM, Kim HD, Lee JS, Slama A. Safe and effective use of the ketogenic diet in children with epilepsy and mitochondrial respiratory chain complex defects. Epilepsia 2007;48:82-8.

17. Lee PR, Kossoff EH. Dietary treatments for epilepsy: management guidelines for the general practitioner. Epilepsy Behav 2011;21(2):115-21.

18. Ramírez-Camacho A, Meavilla S, Catalán N, Gutiérrez A, Campistol J. Experiencia con la dieta cetogénica como tratamiento en la epilepsia refractaria. Rev Neurol 2011;53:524-30.

19. Kinsman SL, Vining EPG, Quaskey SA, Mellits D, Freeman JM. Efficacy of the ketogenic diet for intractable seizure disorders: review of 58 cases. Epilepsia 1992;33:1132-6.

20. Beniczky S, Miranda MJ, Alving J, Heber Povlsen J, Wolf P. Effectiveness of the ketogenic diet in a broad range of seizure types and EEG features for severe childhood epilepsies. Acta Neurol Scand 2010;121:58-62.

21. Raimann X, Marin V. Dieta cetogénica en epilepsia refractaria: Eficacia, evolución y complicaciones a largo plazo. Rev Chil Pediatr 2007;78(5):477-81.

22. Matamala M, Witting S, Troncoso M, et al. Dieta cetogénica: una alternativa eficaz y segura en el tratamiento de epilepsia refractaria en Pediatría. Rev Chil Epilep 2020; 1:17-25.

23. Brooks J, Day S. Low weight, morbidity and mortality in children with cerebral palsy: new Clinical Growth Charts. Pediatrics 2011;128(2):299-306.

24. Patrones de Crecimiento de lactantes, niños y niñas menores de 5 años (OMS 2006). http://www.who.int/childgrowth/ standards/es/ revisada el 4 de abril de 2020.

25. Patrones de Crecimiento de escolares y adolescentes entre 5 años 1 mes y 19 años (OMS 2007). https://www.who.int/ growthref/en/ revisada el 4 de abril de 2020.

26. Zacarías I, Barrios I, González C, Loeff T, Vera G. Tabla de composición de alimentos. INTA - Universidad de Chile, 2018.

27. Cornejo V, Cruchet S. Nutrición en el ciclo vital. 1ª Edición. Santiago, Chile: Editorial Mediterráneo; 2014.

28. Vaccarezza M, Agustinho A. National consensus on the modified Atkins diet.
Rev Neurol 2016;62(8):371-6.

29. Kossoff EH, Auvin S, Ballaban-Gil K, et al.+ Optimal clinical management of children receiving dietary therapies for epilepsy: Updated recommendations of the International Ketogenic Diet Study Group. Epilepsia Open 2018;3(2):175-92.

30. National Cholesterol Education Program (NCEP): highlights of the report of the expert Panel on blood cholesterol levels in children and adolescents. Pediatrics 1992;89:495-501.

31. Expert Panel on integrated guidelines for cardiovascular health and risk reduction in children and adolescents; National Heart, Lung, and Blood Institute: summary report. Pediatrics 2011;128:S213-56.

32. Barja S, Cordero M, Baeza C, Hodgson M. Diagnóstico y tratamiento de las dislipidemias en niños y adolescentes. Recomendaciones de la Rama de Nutrición de la Sociedad Chilena de Pediatría. Rev Chil Pediatr 2014;85(3):367-77.

33. Ko A, Jung DE. The Efficacy of Ketogenic Diet for Specific Genetic Mutation in Developmental and Epileptic Encephalopathy. Front Neurol. 2018;9:530

34. Bramnert M, Segerlantz M, Laurila E, et al. Growth hormone replacement therapy induces insulin resistance by activating the glucose fatty acid cycle. J Clin Endocrinol Metab. 2003;88(4):145563.

35. De Wachter E, Malfroot A, De Schutter I, Vanbesien J, De Schepper J. Inhaled budesonide induced Cushing syndrome in cystic fibrosis patients, due to drug inhibition of cytochrome P450. J Cyst Fibros 2003;2(2):72-5.

36. Farasat S, Kossoff EH, Pillas DJ, et al. The importance of parental expectations of cognitive improvement for their children with epilepsy prior to starting the ketogenic diet. Epilepsy Behav 2006;8(2):406-10.

37. Abend NS, Dlugos DJ. Treatment of refractory status epilepticus: literature review and a proposed protocol. Pediatr Neurol 2008;38(6):377-90.

38. Lin JJ, Lin KL, Chan OW, et al. Intravenous ketogenic diet therapy for treatment of the acute stage of Superrefractory Status Epilepticus in a pediatric patient. Pediatr Neurol 2015;52:442-5.

39. Dressler A, Haiden N, TrimmelSchwahofer P, Benninger F, Samueli S, et al. Ketogenic parenteral nutrition in 17 pediatric patients with epilepsy. Epilepsia Open 2018;3(1):30-9.

40. Massino SA. Ketogenic diet and metabolic therapies. Expanded roles in health and disease. New York: Oxford university Press; 2017.
41. Cai QY, Zhou ZJ, Luo R, et al. Safety and tolerability of the ketogenic diet used for the treatment of refractory childhood epilepsy: a systematic review of published prospective studies. World J Pediatr. 2017;13(6):528-36.

42. Martin K, Jackson CF, Levy RG, Cooper $\mathrm{PN}$. Ketogenic diet and other dietary treatments for epilepsy. Cochrane Database Syst Rev. 2016;2:CD001903.

43. Tagliabue A, Bertoli S, Trentani C, Borrelli P, Veggiotti P. Effects of the ketogenic diet on nutritional status, resting energy expenditure, and substrate oxidation in patients with medically refractory epilepsy: a 6-month prospective observational study. Clin Nutr 2012;31:246-9.

44. Armeno M, Araujo C, Sotomontesano B, Caraballo R. Actualización sobre los efectos adversos durante la terapia con dieta cetogénica en la epilepsia refractaria pediátrica. Rev Neurol. 2018;66(6):193200.

45. Nizamuddin J, Turner Z, Rubenstein J, Pyzik P, Kossoff EH. Management and risk factors for dyslipidemia with the ketogenic diet. J Child Neurol. 2008;23:758-61.

46. Rodríguez-Cruz M, Tovar A. Molecular mechanisms of action and health benefits of polyunsaturated fatty acids. Rev Invest clin. 2005;57(3):457-72.

47. Carvajal A, Ortega RM. La dieta mediterranea como modelo de dieta prudente y saludable. Rev Chil Nutr 2001;28(2):224-36.

48. Burlingame B, Nishida C, Uauy R, Weisell R. Fats and fatty acids in human nutrition; FAO/WHO Expert Consultation. Ann Nutr Metab 2009;55:1-3.

49. Vining EP, Pyzik P, McGrogan J, et al. Growth of children on the ketogenic diet. Dev Med Child Neurol. 2002;44:796-802.

50. Armeno M, Verini A, Del Pino M, et al. A prospective study on changes in nutritional status and growth following two years of ketogenic diet (KD) therapy in children with refractory epilepsy. Nutrients. 2019;11(7):1596.

51. Williams S, Basualdo-Hammond C, Curtis R, Schuller R. Growth retardation in children with epilepsy on the ketogenic diet: a retrospective chart review. J Am Diet Assoc. 2002;102:405-7.

52. Peterson SJ, Tangney CC, PimentelZablah EM, Hjelmgren B, Booth G, BerryKravis $\mathrm{E}$. Changes in growth and seizure reduction in children on the ketogenic diet as a treatment for intractable epilepsy. J Am Diet Assoc 2005;105:718-25.

53. Ferraris C, Guglielmetti M, Pasca L, et al. Impact of the Ketogenic Diet on linear growth in children: a singlecenter retrospective analysis of 34 cases. Nutrients 2019;26:11. 